\title{
Concordância parental sobre problemas de comportamento infantil através do CBCL
}

\author{
Juliane Callegaro Borsa \\ Universidade Federal do Rio Grande do Sul, Porto Alegre-RS, Brasil \\ Maria Lucia Tiellet Nunes \\ Pontifícia Universidade Católica do Rio Grande do Sul, Porto Alegre-RS, Brasil
}

\begin{abstract}
Resumo: Este estudo versa sobre a concordância entre respostas de pais e mães relativas aos problemas de comportamento do mesmo filho através do instrumento Child Behavior Checklist [CBCL-6/18]. Participaram desta pesquisa, 146 casais, com filhos de seis a dez anos, não-clínicos, estudantes do ensino fundamental de Porto Alegre-RS, Brasil. Os questionários foram enviados para todos os pais de crianças desta faixa etária. As respostas ao CBCL classificam a criança como Clínica ou Não-Clínica nas escalas: Competência Social, Problemas Internalizantes, Problemas Externalizantes e Problemas Totais de Comportamento. A concordância entre as respostas de pais e mães para os Problemas Internalizantes e Externalizantes foi moderada $(\mathrm{K}=0,464 ; \mathrm{K}=0,572)$; para a Competência Social e Problemas Totais de Comportamento, a concordância foi baixa $(\mathrm{K}=0,327 ; \mathrm{K}=0,347)$. Tais resultados apontam que pais e mães tendem a não concordar quando solicitados a se pronunciar sobre problemas de comportamento dos filhos.
\end{abstract} Palavras-chave: Relações pais-criança. Lista de verificação comportamental para crianças. CBCL. Criança-problema.

\section{Parental agreement regarding children behavioral problems through the CBCL}

\begin{abstract}
This study aimed to verify the agreement level between answers of fathers and mothers related to the behavioral problems of their children through the Child Behavior Checklist 6/18 [CBCL-6/18]. The sampled consisted of 146 couples with non-clinical children aged six to ten years old, students of an elementary school in Porto Alegre, RS, Brazil. Questionnaires were sent to parents of all children in this age range. The parents' answers to the CBCL classify children as Clinical or Non-Clinical in the following scales: Social Competence, Internalizing Problem Behavior, Externalizing Problem Behavior and Total Behavior Problems. Agreement between fathers and mothers answers was moderate for Internalizing and Externalizing problems $(\mathrm{K}=0.464$; $\mathrm{K}=0.572)$ and low for Social Competence and Total Behavior Problems ( $\mathrm{K}=0.327 ; \mathrm{K}=0.347)$. These results confirm findings in the literature, which report little agreement between fathers and mothers reports regarding their children's behavioral problems.
\end{abstract}

Keywords: Parent child relations. Child behavior checklist. CBCL. Problem children.

\section{Concordancia de los padres acerca de los problemas de comportamiento infantil por medio del CBCL}

\begin{abstract}
Resumen: El estudio constató la concordancia entre las respuestas de padres y madres con respecto a los problemas del comportamiento de un mismo hijo a partir del Child Behavior Checklist 6/18 [CBCL-6/18]. Los informantes fueron 146 parejas, con hijos de seis a diez años, no-clínicos, estudiantes de escuelas primarias de Porto Alegre, RS, Brasil. Los instrumentos fueran enviados para todos parejas con hijos con cuesta edad. Las respuestas a lo CBCL indican si el niño es Clínico o No-Clínico en las escalas Competencia Social, Problemas Internalizantes, Problemas Externalizantes y Problemas Totales de Comportamiento. El análisis indicó concordancia moderada para las respuestas en las escalas Internalizante y Externalizante $(K=0,464 ; K=0,572)$. En las escalas Competencia Social y Problemas Totales de Comportamiento la concordancia fue baja $(\mathrm{K}=0,327 ; \mathrm{K}=0,347)$. Los resultados afirman que padres y madres tienden a no concordar cuando se pronuncian acerca de los problemas de comportamiento de sus hijos.
\end{abstract}

Palabras clave: Relaciones padres-niños. Lista de verificación del comportamiento infantil. CBCL. Niño-problema 


\section{Introdução}

Na família ocidental, os papéis de homens e mulheres têm sido tradicionalmente diferentes (Piccinini, Silva, Gonçalves, Lopes, \& Tudge, 2004) e estas diferenças se evidenciam, por exemplo, no âmbito familiar, em que o trabalho doméstico e o cuidado da prole continuam sendo atribuídos à mulher (Rocha-Coutinho, 2003). Esta, por sua vez, sempre ocupou um lugar fundamental na família, através do papel da maternidade (Favaro, 2007); já o homem foi sempre apoiado pela cultura que lhe atribuiu um lugar à parte, no que diz respeito à relação constituída pela mulher e a criança (Gomes \& Resende, 2004).

Na sociedade contemporânea, no entanto, esses papéis vêm sendo modificados, estabelecendo novas formas de relações no contexto sócio-familiar (Negreiros \& Féres-Carneiro 2004). Com a entrada de um número mais expressivo de mulheres no mercado de trabalho e sua maior participação na economia, já é possível perceber um compartilhamento das tarefas referentes à educação da prole e à organização da família (Fleck \& Wagner, 2003; Levandowski, Koller, \& Piccinini, 2002; Wagner, Predebon, Mosmann, \& Verza, 2005).

Essas mudanças, muitas vezes, remetem os homens ao exercício de funções de cuidado com a prole e têm gerado aumento de interesse sobre a paternidade em nível mundial. Esta vertente tem apontando para importância do pai para a família e para o próprio desenvolvimento da criança (Levandowski e cols., 2002; Mora, Otálora, \& Recagno-Puente, 2005). Contudo, apesar das diferentes configurações quanto aos papéis familiares, a tarefa de acompanhar o cotidiano das crianças ainda é atribuída à mãe e não ao pai (Wagner e cols., 2005). Para Rocha-Coutinho (2003), tanto os homens como as próprias mulheres ainda acreditam que a casa e os filhos(as) são responsabilidades da mulher, enquanto que o provimento financeiro da família é responsabilidade do homem.

Embora a participação do pai no contexto familiar esteja mudando, a crença de que os homens são menos capazes de cuidar da prole acaba por exclui-los das tarefas referentes a esses cuidados. Os homens, ainda hoje, continuam sendo considerados e valorizados pelos papéis exercidos fora do âmbito das relações familiares (Lewis \& Dessen, 1999) e essa realidade contribui para que se dediquem menos aos/ às filhos(as) e às atividades domésticas do que as mulheres (Soares \& Sabóia, 2007). Os diferentes lugares ocupados por homens e mulheres e seus diferentes papéis na família, implicarão na maneira como irão interagir, perceber e se pronunciar em relação às suas crianças, sobretudo no que diz respeito às suas características comportamentais e de desenvolvimento.

A tentativa de compreender os papéis de homens e mulheres no âmbito familiar vem crescendo consideravelmente, sobretudo no que diz respeito à relação com os filhos(as). Existe, ainda, a tentativa de compreender as diferenças e semelhanças na maneira com que pais e mães observam, percebem e se pronunciam sobre suas crianças. Comumente estas informações são medidas através de instrumentos eficazes para quantificar as respostas de pais e mães em relação aos/às filhos(as) (Achenbach, McConaughy, \& Howell, 1987; Duhig, Renk, Epstein, \& Phares, 2000; Kerr, Lunkenheimer, \& Olson, 2007).

Um exemplo de instrumento útil para este fim é o Child Behavior Checklist-CBCL, em português denominado Lista de Verificação Comportamental para Crianças ou Adolescentes (Achenbach, 2001). Uma vez que esse instrumento proporciona o cruzamento de respostas de múltiplos informantes, tornase possível verificar o quão concordantes são as respostas fornecidas (Achenbach, 2006; Kerr e cols., 2007).

Estudos sobre as diferenças e semelhanças nas respostas de pais e mães, quando convidados a se pronunciar sobre suas crianças através de instrumentos, apontam para uma concordância de respostas de nível baixo a moderado (Duhig e cols., 2000; Achenbach, 2006). Estes achados sugerem que mesmo que pais e mães convivam com a mesma criança, em um mesmo ambiente, a qualidade da relação parental e a quantidade de tempo que cada um dedica aos filhos(as) são diferentes, o que irá interferir na maneira com que irão perceber e se pronunciar sobre suas crianças.

Segundo Achenbach (1992), as diferenças na percepção de pais e mães podem ocorrer porque as exposições situacionais e os graus de interação parental são percebidos de forma distinta, de acordo com o grau de insight de cada um, resultando em 
diferentes respostas quando solicitados a fornecer informações sobre os filhos(as). As interações vivenciadas por pais e filhos(as) e mães e filhos(as) são únicas, o que viabiliza a cada um destes respondentes fornecer informações adicionais, além dos dados para os quais ambos concordam.

Em um estudo norte-americano realizado por Phares (1997), mães apresentaram maior precisão ao se pronunciar sobre suas crianças quando comparadas aos pais. A hipótese para essa diferença é que elas convivem mais tempo com os filhos(as), estando mais expostas às situações em que o comportamento pode ser observado. Outra hipótese está relacionada às diferentes maneiras com que as crianças tendem a se comportar na presença dos pais ou das mães que, por sua vez, irão observar filhos(as) de maneira distinta (Hay, Sharp, Pawlby, \& Schmücker, 1999). Este dado pode explicar, por exemplo, o fato das mães reportarem mais problemas de comportamento de suas crianças do que os pais (Duhig e cols., 2000). Essa idéia pode estar relacionada às atribuições sociais e culturais de homens e mulheres e corroboram a hipótese de que existem diferenças na maneira que pais e mães interagem e consequentemente percebem suas crianças.

Em um estudo meta-analítico norte-americano realizado por Duhig e cols. (2000), que objetivou analisar artigos sobre diferenças de respostas de pais e mães quanto aos problemas de comportamento infantil aos checklists e inventários, concluiu-se que pais e mães apresentam concordância moderada quanto às suas respostas. Estes achados também são encontrados em outros estudos semelhantes (Achenbach, 1992; Hay e cols., 1999).

A concordância entre respostas de diferentes informantes tende a ser maior quando estes convivem em um mesmo ambiente, pais e mães têm maior concordância nas respostas, em relação às respostas de pais e professores, por exemplo (Achenbach, 2006). Porém, para o autor, pais e mães são pouco concordantes, se levado em conta o fato de se tratar da mesma criança, vista em situações semelhantes, cujos respondentes executam papéis, também, semelhantes. Em outro estudo, Achenbach e cols. (1987) examinaram múltiplas respostas de diferentes infor- mantes, a partir do CBCL, e concluíram que a concordância entre a resposta de pais e mães é moderada.

Os autores Treutler e Epkins (2003) sugerem que pais e mães são os melhores informantes para avaliar problemas emocionais e de comportamento de crianças. Contudo, mães costumam apresentar respostas mais precisas que os pais, quando solicitados a responder sobre os filhos(as). Os pais tendem a perder qualidade em suas respostas, e quando pais e mães respondem sobre os filhos(as) a resposta dada pelas mães é mais confiável que aquela dada pelos pais. No entanto, poucas pesquisas têm sido conduzidas utilizando os pais como informantes (Achenbach e cols., 1987), o que pode ser explicado pelo fato de eles serem comumente mais ausentes que as mães e passarem menos tempo em contato com os(as) filhos(as) (Seiffge-Krenke \& Kollmar, 1998).

Assim, o presente estudo justifica-se pela importância de compreender como pais e mães se pronunciam sobre seus filhos(as), especificamente se ambos concordam quanto a problemas de comportamento de sua prole. Considerando que são os pais e mães que trazem as crianças para atendimento psicológico, torna-se importante compreender de que maneira percebem o comportamento delas, abordando as possíveis diferenças nessas percepções. Desta forma, o objetivo do estudo foi verificar a concordância entre as respostas de pais e mães quanto aos problemas de comportamento de seus filhos(as), a partir do CBCL.

\section{Método}

\section{Delineamento}

Este trabalho se constitui em uma pesquisa quantitativa, descritiva, transversal, de medida única, para avaliar a concordância entre pais e mães (casais) com filhos entre seis e dez anos de idade.

\section{Participantes}

Participaram do estudo 146 casais, com filhos de seis a dez anos, meninos e meninas, matriculados em escolas de ensino fundamental do bairro Petrópolis, na cidade de Porto Alegre-RS. O convite para participar do estudo foi enviado a todos os pais $\mathrm{e}$ 
mães dos estudantes das respectivas escolas que, por sua vez, atendiam à faixa etária delimitada para o presente estudo. Para a presente amostra, foram considerados todos os instrumentos devolvidos e devidamente preenchidos. O tamanho da amostra foi calculado por meio do software BioEstat. 4.0 (Programa Epi Info ${ }^{\mathrm{TM}}$ Version 3.3.2, Epi Info ${ }^{\mathrm{TM}}$.), adotando-se um nível de significância de 0,05 e um poder de teste de 0,80 , levando em consideração o número de itens do CBCL e o número de possibilidades de resposta para cada item.

\section{Critérios de inclusão e exclusão para a amostra}

Para o presente estudo foram incluídos apenas os questionários cujos respondentes atendiam às seguintes exigências: ser pais e mães biológicos, padrastos e madrastas ou pais e mães adotivos. Foram excluídos, ainda, os questionários de dez desses casais que obtiveram nível de concordância igual a 1.00, ou seja, que responderam igualmente a todos os itens do instrumento. Esta decisão teve como objetivo minimizar o risco de cópia no tocante ao preenchimento do instrumento, uma vez que o mesmo não foi controlado. Por fim, foram excluídos dois questionários de um casal, em que um dos respondentes pontuou escore dois (muito verdadeiro ou freqüentemente verdadeiro) para todos os itens. Supôs-se que tal instrumento foi preenchido erroneamente ou de forma pouco realista no tocante às características de comportamento da criança Foram considerados, então, para a amostra, 292 questionários de 146 casais $(n=146)$, representados por ser mãe (biológica, adotiva ou madrasta) ou por ser pai (biológico, adotivo ou padrasto) conforme é apresentado na Tabela 1 .

Tabela 1

Número de respondentes ao CBCL

\begin{tabular}{lcc}
\hline \multicolumn{1}{c}{ Respondente } & n & \% \\
\hline Madrasta & 1 & 0,34 \\
Mãe adotiva & 2 & 0,68 \\
Pai adotivo & 4 & 1,37 \\
Padrasto & 5 & 1,71 \\
Pai biológico & 137 & 46,92 \\
Mãe biológica & 143 & 48,98 \\
Total & 292 & 100 \\
\hline
\end{tabular}

\section{Instrumento}

Para a pesquisa foi utilizado o instrumento Child Behavior Checklist (CBCL), destinado à faixa etária de seis a dezoito anos (Achenbach, 2001). Este instrumento foi traduzido para o português como Lista de Verificação Comportamental para Crianças ou Adolescentes (Santos \& Silvares, 2006).

O CBCL 6/18 anos é um questionário composto de 138 itens, destinado aos pais/mães ou cuidadores para que forneçam respostas referentes aos aspectos sociais e comportamentais de seus filhos(as). Do total de itens, 20 são destinados à avaliação da competência social da criança e 118 relativos à avaliação de seus problemas de comportamento. Os itens do questionário listam uma série de comportamentos desejáveis e disruptivos e, para cada um deles, o respondente deve marcar a freqüência com que esses problemas de comportamento ocorrem. Atribui-se a cada item/problema ' 0 ', quando não é verdadeiro; '1', se é um pouco verdadeiro ou às vezes verdadeiro; e ' 2 ', se é muito verdadeiro ou freqüentemente verdadeiro (Achenbach, 2001; Bordin, Mari, \& Caeiro, 1995; Santos \& Silvares, 2006).

Os itens apresentados no CBCL irão compor as onze escalas individuais que correspondem a diferentes problemas de comportamento da criança. Dentre essas escalas, três referem-se à competência social, relativas a problemas no desempenho de atividades $\mathrm{e}$ nos aspectos relacionados à sociabilidade e à escolaridade. A soma dessas escalas origina a Escala de Competência Social.

A Escala de Competência Social é composta por 20 itens relativos ao envolvimento da criança em diversas atividades (brincadeiras, jogos, execução de tarefas), participação em grupos, relacionamento com pessoas (familiares, amigos), independência no brincar e desempenho escolar. (Bordin e cols., 1995). A maioria dos itens exige que os pais comparem o comportamento do filho com outras crianças da mesma idade, identificando-as como Abaixo da Média, Acima da Média ou Dentro da Média (Achenbach, 1991).

As outras oito escalas são de Ansiedade/Depressão, Isolamento/Depressão, Queixas Somáticas, 
Problemas Sociais, Problemas de Pensamento, Problemas de Atenção, Comportamento de Quebrar Regras/Delinqüencial e Comportamento Agressivo, cuja soma dá origem à Escala Total de Problemas de Comportamento (Achenbach, 1991; Massola \& Silvares, 2005; Silvares, Meyer, Santos, \& Gerencer, 2006).

A Escala Total de Problemas de Comportamento é constituída, ainda, pela Escala de Problemas de Comportamento Internalizante e pela Escala de Problemas de Comportamento Externalizante. A Escala de Problemas de Comportamento Internalizante corresponde às três primeiras escalas de problemas de comportamento: (1) ansiedade e depressão; (2) isolamento e depressão e (3) queixas somáticas. A Escala de Problemas de Comportamento Externalizante corresponde às duas últimas escalas de problemas de comportamento: Comportamento de Quebrar Regras e Comportamento Agressivo (Santos \& Silvares, 2006).

A Escala de Problemas de Comportamento Externalizante é descrita em termos de padrões comportamentais manifestos e desajustados, denominados também de problemas de comportamento, como agressividade, agitação psicomotora e comportamento delinqüente. Refere-se, em geral, aos comportamentos considerados problemáticos, que se exercem diretamente sobre o ambiente. A Escala de Problemas de Comportamento Internalizante é descrita em termos de padrões comportamentais privados desajustados, denominados também de problemas emocionais, como tristeza e isolamento. Esta, por sua vez, refere-se a um conjunto de comportamentos considerados problemáticos pelos entrevistados, mas que não exercem diretamente sobre o ambiente, restringindo-se ao mundo interno da criança. Ambas as escalas, Internalizante e Externalizante, compõem a Escala Total de Problemas de Comportamento (Bordin e cols., 1995; Gauy \& Guimarães, 2006; Massola \& Silvares, 2005).

A Escala Total de Problemas de Comportamento também é composta pelas escalas Problemas Sociais, Problemas de Pensamento e Problemas de Atenção, que não pertencem à Escala Externalizante e à Escala Internalizante. Ainda na composição da Escala Total de Problemas de Com- portamento inclui-se uma categoria denominada Outros Problemas, que correspondem a alguns itens não englobados em nenhuma das escalas anteriores. No entanto, todos os itens de Outros Problemas, somados aos demais itens das outras escalas, são utilizados para calcular o escore de problemas totais de comportamento, fornecendo, assim, a Escala Total de Problemas de Comportamento.

Em todas as 11 escalas do CBCL, a criança é classificada, conforme propõe o instrumento, como Clínica, Limítrofe ou Não-Clínica, de acordo com a amostra normativa de pares de Achenbach (1991). Dependendo dos objetivos do estudo, as categorias de classsificação dos resultados do CBCL podem ser reduzidas em duas: Clínica e Não-Clínica, através da inclusão dos casos Limítrofes na categoria Clínica (Achenbach, 1991). Essa classificação não representa, contudo, um diagnóstico da criança; aponta, apenas, a categoria na qual a criança melhor é classificada, de acordo com o instrumento.

\section{Procedimentos}

\section{Coleta de dados}

Primeiramente foi realizado contato telefônico com a Secretaria da Educação do Estado do Rio Grande do Sul, que forneceu, via e-mail, uma lista atualizada das escolas localizadas no bairro Petrópolis de Porto Alegre-RS. A escolha por escolas desse bairro ocorreu por conveniência, considerando o grande número de escolas na região e a localização acessível à pesquisadora principal e a outros auxiliares da pesquisa.

Após o recebimento da lista das escolas, foi realizado o contato com as mesmas, também por telefone, para explicar a pesquisa e saber do interesse na participação do estudo. Para as escolas interessadas, foram solicitadas informações sobre o número de crianças matriculadas, faixa etária e número de turmas. Em um segundo momento, foi entregue a cada escola uma cópia resumida do projeto de pesquisa, uma carta de apresentação e uma carta de aceite em participar da pesquisa.

Com o aceite das escolas em participar do estudo, foi realizado o contato com os pais. A entrega do instrumento CBCL para os pais foi realizada pelas 
crianças através de um envelope lacrado. Ao todo, foram entregues 400 envelopes, ou seja, para 400 casais participantes. $\mathrm{O}$ envelope continha a Carta de Apresentação da pesquisa, o Termo de Consentimento Livre e Esclarecido, uma folha explicativa quanto aos procedimentos de preenchimento do CBCL e o CBCL propriamente dito. Aos pais foi solicitado que respondessem ao instrumento separada e individualmente, considerando o(a) mesmo(a) filho(a) e, depois de preenchido, que o reenviassem à escola, dentro de outro envelope, devidamente lacrado. Foi enfatizada aos pais a importância dos questionários serem respondidos individualmente, não havendo, assim, a comunicação entre os respondentes.

\section{Análise de dados}

As respostas dos pais e das mães aos itens do CBCL foram analisadas a partir do Software Assessment Data Manager (ADM), que é o programa desenvolvido para correção do CBCL. Esse programa é o software central do Sistema de Avaliação Empiricamente Baseado de Achenbach (Achenbach System of Empirically Based Assessment - ASEBA) e é utilizado para a análise de todos os questionários do ASEBA, dentre eles o CBCL 6/18. O programa inclui módulos para digitar e analisar os dados obtidos através deste instrumento (Achenbach \& Rescorla, 2004).

Conforme mencionado, o programa ADM, ao corrigir as respostas fornecidas aos itens/problemas do CBCL, classifica a criança a partir das categorias Clínica, Limítrofe e Não-Clínica (Achenbach, 1991). Em outras palavras, as respostas fornecidas por pais e mães aos itens/problemas são alocadas nessas categorias. O presente estudo optou por incluir as crianças categorizadas como Limítrofes na categoria Clínica, conforme recomendação de Achenbach, para pesquisas com o CBCL.

Os resultados do CBCL oferecidos pelo ADM foram analisados através do programa estatístico SPSS for Windows, versão 11. Foram calculadas as médias, freqüências e porcentagens relativas às respostas fornecidas pelos pais e pelas mães ao instrumento CBCL. A análise de concordância entre as respostas dos casais, a partir da classificação das crianças em Clínica e Não-Clínicas foi realizada através da medida Kappa. Para análise deste índice seguiu-se a classificação de Landis \& Koch (1977): Kappa $<0=$ Sem Concordância; Kappa entre 0 e 0.19=Concordância Pobre; Kappa entre 0.20 e 0.39 =Concordância Baixa; Kappa entre 0.40 e 0.59=Concordância Moderada; Kappa entre 0.60 e 0.79=Concordância Substancial; Kappa entre 0.80 e 1.00=Concordância Quase Perfeita.

\section{Considerações éticas}

Inicialmente, foi entregue às escolas um conjunto de materiais constituído pelos seguintes documentos: (1) cópia resumida do projeto, explicando todos os procedimentos e objetivos da pesquisa; (2) Carta de Apresentação da Pesquisa; (3) Termo de Consentimento Livre e Esclarecido, o qual foi assinado pelas diretoras das escolas e devolvido às pesquisadoras. Após o aceite das escolas, foi entregue aos pais o Termo de Consentimento Livre e Esclarecido, o qual foi assinado e devolvido à pesquisadora. Este estudo foi aprovado pela Comissão Científica da Faculdade de Psicologia da Pontifícia Universidade Católica do Rio Grande do Sul (PUCRS) e pelo Comitê de Ética em Pesquisa da mesma instituição.

\section{Discussão}

O objetivo de verificar a concordância entre respostas de pai e mãe de uma mesma criança, de acordo com o CBCL, foi examinado a partir da medida Kappa. As respostas de pai e mãe da mesma criança para as Escalas de Competência Social, Problemas de Comportamento Internalizante, Problemas de Comportamento Externalizante e Problemas Totais de Comportamento foram calculadas pelo ADM, sendo a criança classificada em Clínica ou Não-Clínica.

\section{Escala de competência social}

A Escala de Competência Social é composta por 20 itens relativos ao envolvimento da criança em diversas atividades (brincadeiras, jogos, execução de tarefas), participação em grupos, relacionamento com pessoas (familiares, amigos), independência no brincar e desempenho escolar (Bordin e cols., 1995). 
A análise das respostas de pais e mães para a Escala de Competência Social, através da medida Kappa, resultou em uma concordância baixa ( $K=0,327 ; p=0,001)$, ou seja, pais tendem a não concordar em suas respostas quanto aos problemas relacionados à competência social dos seus filhos(as). A Tabela 2 apresenta a distribuição das respostas dos casais na escala referente aos problemas de competência social, considerando a classificação que a própria escala fornece, em termos de ser a criança Clínica ou Não-Clínica.

Tabela 2

Concordância entre pais e mães na Escala de Competência Social

\begin{tabular}{|c|c|c|c|c|c|}
\hline & & \multicolumn{4}{|c|}{$\begin{array}{c}\text { Problemas de } \\
\text { competência social } \\
\text { percebidos pelo pai }\end{array}$} \\
\hline & & \multicolumn{2}{|c|}{ Clínico } & \multicolumn{2}{|c|}{ Não-clínico } \\
\hline & & n & $\%$ & $\mathbf{n}$ & $\%$ \\
\hline $\begin{array}{l}\text { Problemas de } \\
\text { competência } \\
\text { social }\end{array}$ & Clínico & 32 & 29,1 & 14 & 12,7 \\
\hline $\begin{array}{l}\text { percebidos pela } \\
\text { mãe }\end{array}$ & Não-clínico & 23 & 20,9 & 41 & 37,3 \\
\hline Total & & 55 & 50 & 55 & 50 \\
\hline
\end{tabular}

Clínico = Crianças classificadas como Clínicas, de acordo com o CBCL, quanto aos problemas de competência social.

Não-Clínico = Crianças classificadas como Não-Clínicas, de acordo com o CBCL, quanto aos problemas de competência social.

Tamanho da amostra calculado por meio do software BioEstat. 4.0 (Programa Epi Info ${ }^{\mathrm{TM}}$ Version 3.3.2, Epi Info ${ }^{\mathrm{TM}}$.)

A análise da Escala de Competência Social foi realizada com os questionários preenchidos por 55 pais e 55 mães, considerando que 36 casais preencheram os questionários de forma incompleta nos itens relativos ao desempenho escolar. A maioria destas 36 crianças são alunos de primeira série que não possuem em sua grade curricular todas as disciplinas citadas no questionário CBCL.

Escala de Problemas de Comportamento Internalizante

Conforme já referido, a Escala de Problemas de Comportamento Internalizante corresponde aos problemas de comportamentos mais privados da criança, como tristeza e isolamento. A análise das respostas de pais e mães para a Escala de Problemas de Comportamento Internalizante, através da medida Kappa, resultou em uma concordância moderada $(K=0,464 ; p<0,000)$, ou seja, a concordância entre respostas de pais e mães não é considerada substancial no que diz respeito aos problemas de comportamento internalizante de seus filhos. A Tabela 3 apresenta a distribuição das respostas dos casais na escala referente aos problemas de comportamento internalizante, respeitando novamente a classificação da criança em Clínica ou Não-Clínica, conforme o próprio instrumento.

Tabela 3

Concordância entre pais e mães para Escala de Problemas de Comportamento Internalizante

Problemas de

comportamento

internalizante

percebidos pelo pai

Clínico Não-clínico

\begin{tabular}{lccccc} 
& & $\mathbf{n}$ & $\boldsymbol{\%}$ & $\mathbf{n}$ & $\boldsymbol{\%}$ \\
\hline $\begin{array}{l}\text { Problemas de } \\
\begin{array}{l}\text { comportamento } \\
\text { internalizante } \\
\text { percebidos pela }\end{array}\end{array}$ & Clínico & 37 & 25,3 & 23 & 15,8 \\
mãe & Não-clínico & 14 & 9,6 & 72 & 49,3 \\
Total & & 51 & 34,9 & 95 & 65,1 \\
\hline
\end{tabular}

Clínico = Crianças classificadas como Clínicas, de acordo com o CBCL, quanto aos problemas de comportamento internalizante. Não-Clínico = Crianças classificadas como Não-Clínicas, de acordo com o CBCL, quanto aos problemas de comportamento internalizante.

\section{Escala de Problemas de Comportamento Externalizante}

A Escala de Problemas de Comportamento Externalizante corresponde aos problemas de comportamento desajustados que se exercem diretamente no ambiente. A análise das respostas de pais e mães para a Escala de Problemas de Comportamento Externalizante, através da medida Kappa, resultou em uma concordância moderada $(K=0,572 ; p<0,000)$, ou seja, a concordância entre respostas de pais e mães 
não é considerada substancial no que diz respeito aos problemas de comportamento externalizante de seus filhos. A Tabela 4 apresenta a distribuição das respostas dos casais na escala referente aos problemas de comportamento externalizante, classificando as crianças em Clínicas ou Não-Clínicas, conforme o instrumento.

Tabela 4

Concordância entre pais e mães para Escala de Problemas de Comportamento Externalizante

Problemas de comportamento externalizante percebidos pelo pai Clínico Não-clínico

\begin{tabular}{lccccc} 
& & n & $\%$ & n & $\%$ \\
\hline $\begin{array}{l}\text { Problemas de } \\
\begin{array}{l}\text { comportamento } \\
\text { externalizante } \\
\text { percebidos pela } \\
\text { mãe }\end{array}\end{array}$ & Clínico & 24 & 16,4 & 13 & 8,9 \\
$\begin{array}{l}\text { Total } \\
\text { No-clínico }\end{array}$ & 10 & 6,9 & 99 & 67,8 \\
\hline
\end{tabular}

Clínico = Crianças classificadas como Clínicas, de acordo com o CBCL, quanto aos problemas de comportamento externalizante. Não-Clínico = Crianças classificadas como Não-Clínicas, de acordo com o CBCL, quanto aos problemas de comportamento externalizante.

\section{Escala Total de Problemas de Comportamento}

A Escala Total de Problemas de Comportamento é a soma das Escalas de Problemas de Comportamento Internalizante e Externalizante. A estas devem ser adicionados os resultados nas escalas Problemas Sociais, Problemas de Atenção e Problemas de Pensamento, além dos itens denominados Outros Problemas.

A análise das respostas de pais e mães para a Escala Total de Problemas de Comportamento, através da medida Kappa, resultou em uma concordância baixa $(K=0,347$; $<<0,000)$. Pode-se dizer, portanto, que os pais tendem a não concordar em suas respostas quanto aos problemas totais de comportamento de seus filhos(as). A Tabela 5 apresenta a distribuição das respostas dos casais na Escala Total de Problemas de Comportamento, mais uma vez pela classificação Clínica ou Não-Clínica, resultante do próprio instrumento.

Tabela 5

Concordância de pais e mães para Escala Total de Problemas de Comportamento

\begin{tabular}{|c|c|c|c|c|c|}
\hline & & \multicolumn{4}{|c|}{$\begin{array}{l}\text { Problemas totais de } \\
\text { comportamento } \\
\text { percebidos pelo pai }\end{array}$} \\
\hline & & \multicolumn{2}{|c|}{ Clínico } & \multicolumn{2}{|c|}{ Não-clínico } \\
\hline & & $\mathbf{n}$ & $\%$ & $\mathbf{n}$ & $\%$ \\
\hline $\begin{array}{l}\text { Problemas } \\
\text { totais de } \\
\text { comportamento } \\
\text { percebidos pela } \\
\text { mãe }\end{array}$ & Não-clínico & 28 & 19,2 & 27 & 18,5 \\
\hline Total & & 44 & 30,1 & 102 & 69,9 \\
\hline
\end{tabular}

Como foi possível observar, a concordância entre as respostas de pais e mães para os Problemas Internalizantes e para os Problemas Externalizantes foi moderada ( $K=0,464$ e $K=0,572$, respectivamente). Em relação aos Problemas Competência Social e Problemas Totais de Comportamento, a concordância entre as respostas foi baixa $(\mathrm{K}=0,327$ e $\mathrm{K}=0,347)$. Em outras palavras, observa-se que a concordância entre as respostas de pais e mães às quatro escalas CBCL, que quando calculadas classificam a criança como Clínica ou Não-Clínica, foi de baixa a moderada.

Uma hipótese para o fato de pais e mães terem obtido baixa concordância entre si para a Escala de Competência Social é que tal competência da criança é exercida em diversos ambientes, nos quais os pais nem sempre estão tão presentes, como é o caso da escola, dentre outros. O conceito de Competência Social advém da teoria comportamental e refere-se a determinados tipos de comportamentos sociais, caracterizados pela capacidade que o indivíduo tem de organizar pensamentos, sentimentos e ações, em função de objetivos pessoais e sociais, em 
prol de consequiências positivas, sobretudo nas relações interpessoais estabelecidas por este sujeito. A competência social de crianças refere-se ao rendimento escolar, às estratégias de enfrentamento diante de situações de estresse ou frustração, ao autocuidado, à independência para realizar tarefas, à cooperação, entre outras habilidades (Cia, Pamplin, \& Del Prette 2006).

Os estudos sobre concordância parental a partir do CBCL com o objetivo de verificar a concordância de respostas de diferentes informantes ao CBCL, comumente não analisam as respostas referentes à Escala de Competência Social. Esses estudos detêm-se nas Escalas de Problemas de Comportamento Internalizante e Externalizante, bem como a Escala Total de Problemas de Comportamento. No estudo meta-analítico realizado por Duhig e cols. (2000), foram analisados 60 artigos publicados entre os anos de 1990 e 1997 que possuíam dados quantitativos referentes às respostas de pais e mães acerca dos problemas comportamentais e emocionais de crianças e adolescentes, a partir de questionários e checklists. Os problemas de comportamento analisados nos artigos citados foram classificados em Externalizantes, Internalizantes e Problemas Totais de Comportamento; a Escala de Competência Social, por sua vez, não foi analisada. Em função dessa realidade, ao menos neste momento, não foi possível realizar uma análise das respostas fornecidas pelos casais deste estudo à luz da literatura.

A concordância moderada entre as respostas de pais e mães para a Escala de Problemas de Comportamento Internalizante e para a Escala de Problemas de Comportamento Externalizante, assim como a baixa concordância na Escala Total de Problemas de Comportamento, indica que mesmo que pais e mães estejam se pronunciando sobre a mesma criança, as suas características de comportamento, quais sejam, são observadas de forma distinta. É possível compreender esses achados à luz dos estudos que sugerem ser diferente tanto a qualidade da relação parental como a quantidade de tempo que pais e mães dedicam aos filhos(as), o que irá interferir na maneira com que irão perceber e se pronunciar sobre suas crianças (Achenbach, 1992; Duhig e cols., 2000; Achenbach, 2006).

Outra hipótese para esse fenômeno se dá pelas características da família contemporânea cujas crianças passam pouco tempo em contato com os pais e mães, que em função do trabalho e outras atividades, deixam, muitas vezes, a criança aos cuidados de terceiros (De Marque, 2006). O fato de pais e mães passarem pouco tempo em contato com seus filhos(as) pode interferir na maneira que percebem e se pronunciam sobre esses filhos(as).

Especificamente quanto aos problemas de comportamento internalizante, a literatura aponta que esses são pouco perceptíveis por parte daqueles que convivem com a criança. Esses problemas acontecem mais no âmbito privado da criança e geralmente pais e mães têm dificuldade em percebê-los e, por conseguinte, reportá-los (Achenbach e cols., 1987; Duhig e cols., 2000; Kerr e cols., 2007). Essas características podem ser responsáveis por uma maior concordância nas respostas de pais e mães para os problemas de comportamento externalizante, em detrimento dos problemas de comportamento internalizante (Achenbach e cols., 1987; Duhig e cols., 2000; Kerr e cols., 2007). Contudo, no presente estudo a concordância das respostas de pais e mães nas escalas internalizante e externalizante, foi moderada.

Por fim, os achados deste estudo corroboram os estudos internacionais sobre a concordância entre respostas de pais e mães, quando convidados a responder sobre os problemas de comportamento de seus filhos. Vários estudos que utilizam o CBCL, bem como outros instrumentos para comparar respostas de informantes, apontam para um nível de concordância baixo a moderado entre as respostas fornecidas por pais e mães (Achenbach, 1992, 2006; Duhig e cols., 2000).

Este é o primeiro estudo brasileiro sobre a concordância de respostas de pais e mães quanto aos 
problemas de comportamento de um mesmo filho(a), a partir do CBCL, considerando que não foi encontrada nenhuma referência sobre estudos semelhantes nas bases de dados pesquisadas ao longo deste estudo (SciELO, BVS-Psi e Portal CAPES). Por outro lado, este estudo apresenta resultados que permitem inferir que os pais e mães desta pesquisa também apresentam percepções distintas entre si, e, por conseguinte, baixa concordância em relação aos problemas de comportamento de seus filhos(as). Aqui, torna-se relevante considerar que a baixa concordância entre as respostas de pais e mães para os problemas de comportamento de um mesmo filho(a) foi levantada a partir dos resultados obtidos através do CBCL. Resultados diferentes podem ser obtidos a partir de outras metodologias ou de outros instrumentos.

\section{Considerações finais}

A presente pesquisa teve como objetivo verificar a concordância entre as respostas de pais e mães quanto aos problemas de comportamento de seus filhos(as), a partir do CBCL. Atingida esta meta, é possível tecer algumas considerações reflexivas sobre os dados apresentados, pensando-se na contribuição em apontar caminhos para novas investigações e questionamentos nesta área.

De acordo com os resultados, verificou-se que pais e mães apresentam nível de concordância baixo a moderado em suas respostas, quando solicitados a se pronunciar sobre os problemas de comportamento de seus filhos, a partir do Child Behavior Checklist, CBCL. Os achados trazidos na presente pesquisa corroboram os achados da literatura internacional, que também apontam concordância baixa a moderada quanto às respostas fornecidas por pais e mães a esse instrumento (Achenbach e cols., 1987; Duhig e cols., 2000; Kerr e cols., 2007).

A baixa concordância entre as respostas fornecidas por pais e mães ao CBCL indica que mesmo que pais e mães estejam em contato com a mesma criança, cada um pode proporcionar informações distintas sobre os problemas de comportamento de seus filhos(as). Inúmeros fatores podem contribuir para essas diferenças. Dentre estes fatores estão os aspectos da relação conjugal e, ainda, outras características subjetivas dos pais e mães. Da mesma forma, parece haver uma influência importante dos aspectos sociais e culturais intrínsecos na definição dos papéis parentais que, por sua vez, irão influenciar o lugar que pais e mães ocuparão no âmbito familiar e, conseqüentemente, na maneira com que irão observar, perceber, se relacionar e conseqüentemente, se pronunciar sobre seus filhos(as) (Achenbach, 1992; Hay e cols., 1999; Seiffge-Krenke \& Kollmar, 1998; Treutler \& Epkins, 2003).

As diferentes formas com que pais e mães percebem seus filhos(as) apresentam profundas implicações, por exemplo, no que diz respeito às queixas dos pacientes infantis trazidos para atendimento psicológico. Já na primeira entrevista, pais e mães tendem a apresentar opiniões diferentes quanto às queixas de seus filhos(as). Isso ocorre devido às diferentes relações estabelecidas entre pais e filhos(as) e mães e filhos(as). Ora, uma vez que pais e mães exercem suas funções parentais de forma qualitativa e quantitativamente distintas, é compreensível que cada um irá observar e perceber os problemas apresentados pela criança também distintamente, apontando diferentes concepções sobre a urgência e a relevância do encaminhamento para a psicoterapia.

Ao mesmo tempo, uma vez discordantes no que diz respeito à gravidade dos problemas apresentados pelos filhos(as), é provável que pais e mães também estejam discordantes quanto à aderência e à manutenção da psicoterapia do filho(a), tanto quanto à sua própria contribuição e participação no processo terapêutico. Em outras palavras, esse contexto implica na qualidade da terapia, no nível de aderência e na taxa de abandono dos pacientes infantis (Kazdin, 1991; Kazdin, Siegel, \& Bass, 1990). 
Devido à relevância da participação de pais e mães no processo terapêutico da criança, considerando que são eles que trazem as crianças para atendimento psicológico e que, por sua vez, são responsáveis pela manutenção do mesmo, torna-se importante compreender de que maneira pais e mães percebem o comportamento das suas crianças, abordando as possíveis diferenças nessas percepções. Nesse contexto, o CBCL pode ser um instrumento útil para tal finalidade.

Quanto ao instrumento, observou-se, no decorrer da pesquisa, que o CBCL possui algumas limitações, conforme segue. Determinados itens podem não corresponder, exatamente, ao que os respondentes denominam problemas de comportamento. Como exemplo, cita-se o item n". 7, "É vaidoso", que os respondentes brasileiros não entenderam como uma característica prejudicial do comportamento da criança. Esta incoerência quanto ao significado do que é considerado, efetivamente, um problema de comportamento, pode ter ocorrido em função da tradução dos itens do instrumento. Quanto à forma de apresentação do instrumento (letras pequenas e apertadas), verificou-se um prejuízo quanto ao correto preenchimento de alguns itens, por parte de alguns casais, o que inviabilizou o uso destes questionários. Ainda, observou-se que algumas informações referentes à competência social da criança não estão de acordo com a realidade das crianças brasileiras, o que pode ter impedido o preenchimento destes itens pelos pais/ mães, comprometendo sua adequada análise. Por fim, entende-se que as categorias de classificação proposta por Achenbach (2001), para os problemas de comportamento das crianças a partir do CBCL não comportam alguns indicadores freqüentes entre crianças brasileiras como é o caso dos problemas de aprendizagem e problemas familiares. Devido ao interesse de sanar tais problemas, estão sendo realizadas constantes reformulações no instrumento, considerando que o mesmo ainda está em fase de padronização no Brasil.
O presente estudo não encerra as diversas questões referentes ao tema discutido aqui. Não foram considerados, por exemplo, os aspectos sociais, sócio-demográficos e econômicos relativos aos sujeitos da presente pesquisa. Do mesmo modo, não foram analisados os aspectos pessoais e subjetivos de pais e mães como, por exemplo, a presença de transtornos emocionais e o nível de satisfação conjugal.

Considerando que foram utilizados os questionários respondidos por pais e mães biológicos, padrastos e madrastas e pais e mães adotivos, casados ou separados, não se sabe quais as possíveis implicações dessas condições na maneira com que cada pai ou mãe irá perceber e se pronunciar sobre seus filhos(as). É importante referir que não foi objetivo deste estudo analisar os aspectos referentes às diferentes configurações familiares e suas respectivas implicações na percepção e nas respostas fornecidas por homens e mulheres em relação aos seus filhos. Entende-se que esses aspectos, além de outras características familiares subjacentes, poderiam ter fornecido informações adicionais importantes para a compreensão do fenômeno em questão.

Por fim, este estudo objetivou verificar o nível de concordância de respostas de pais e mães ao CBCL. Foi solicitado aos respondentes que preenchessem ao questionário individualmente e que não comunicassem os resultados um ao outro. No entanto, os questionários foram entregues em um envelope e não houve controle quanto à troca de informações entre os casais. Devido ao contrato e às recomendações de preenchimento, acredita-se que os respondentes atenderam à solicitação da pesquisadora para responderem aos instrumentos individual e separadamente. Todos os instrumentos com índice de correlação igual a 1,00 (de acordo com o CBCL) foram excluídos.

Neste sentido, sugere-se, portanto, estudos posteriores que objetivem analisar as diferenças e concordâncias entre as respostas de pais e mães em função de características sócio-econômicas, idade das crianças e dos respondentes, bem como avaliar as características conjugais e pessoais dos respondentes. 


\section{Referências}

Achenbach T. M. (1991). Manual for the Child Behavior Checklist/4-18 and 1991 profile. Burlington, VT: University of Vermont.

Achenbach T. M. (1992). Manual for the Child Behavior Checklist/2-3 and 1992 profile. Burlington, VT: University of Vermont.

Achenbach T. M. (2001). Manual for the Child Behavior Checklist/6-18 and 2001 profile. Burlington: University of Vermont.

Achenbach, T. M. (2006). As others see us - clinical and research implications of cross-informant correlations for psychopathology. Current Directions in Psychological Science, 15(2), 94-98.

Achenbach, T. M., McConaughy, S. H., \& Howell, C. T. (1987). Child/adolescent behavioral and emotional problems: Implications of crossinformant correlations for situacional specificity. Psychological Bulletin, 101, 213-232.

Achenbach T. M., \& Rescorla, L. A. (2004). Mental health practitioners' guide for the Achenbach of Empirically Based Assessment (4th ed.). Burlington, VT: University of Vermont, Research Center for Children, Youth, \& Families.

Bordin, I. A. S., Mari, J. J., \& Caeiro, M. F. (1995). Validação da versão brasileira do Child Behavior Checklist [CBCL] - Inventário de comportamento da infância e da adolescência: Dados preliminares. Revista ABP - APAL, 17(2), 55-66.

Cia, F., Pamplin, R. C. O., \& Del Prette, Z. A. P. (2006). Comunicação e participação pais-filhos: Correlação com habilidades sociais e problemas de comportamento dos filhos. Paidéia (Ribeirão Preto), 16, 395-408.

De Marque, C. R. (2006). Construção de identidade e formação de vínculos, no processo psicoterapêutico de uma criança, em diferentes contextos familiares. Dissertação de mestrado não-publicada, Universidade de São Paulo, São Paulo, SP.
Duhig, A. M., Renk, K., Epstein, M. K., \& Phares, V. (2000). Interparental agreement on internalizing, externalizing and total behavior problems: a metaanalysis. Clinical Psychology: Science and Pratice, 7, 435-453.

Favaro, C. (2007). Mulher e família: Um binômio (quase) inseparável. In M. N. Strey; J. A. S. Neto \& R. L. Horta (Orgs.), Família e gênero (pp. 3956). Porto Alegre: EdiPUCRS.

Fleck, A. C., \& Wagner, A. (2003). A mulher como a principal provedora do sustento econômico familiar. Psicologia em Estudo, 8 [No. especial], 31-38.

Gauy, F. V., Guimarães, S. S. (2006). Triagem em saúde mental infantil. Psicologia: Teoria e Pesquisa, 22, 05-16.

Gomes, A. J. S., \& Resende, V. R. (2004). O pai presente: $\mathrm{O}$ desvelar da paternidade em uma família contemporânea. Psicologia: Teoria e Pesquisa, 20, 119-125.

Hay, D. F., Sharp, D., Pawlby, S., \& Schmücker, G. (1999). Parent's judgements about young children's problems: Why mothers and fathers might disagree yet still predict later outcomes. Journal of Child Psychology and Psychiatry, 40, 1249-1258.

Kazdin A. E. (1991). Effectiveness of psychotherapy with children and adolescents. Journal of Counsulting and Clinical Psychology, 59, 785-798.

Kazdin, A. E., Siegel, T. C., \& Bass, D. (1990). Drawing upon clinical practice to inform research on child and adolescent psychotherapy: A survey of practioners. Professional Psychology: Research and Practice, 21, 189-198.

Kerr, D. C. R., Lunkenheimer, E. S., \& Olson, S. L. (2007). Assessment of child problem behaviors by multiple informants: Alongitudinal study from preschool to school entry. Journal of Child Psychology and Psychiatry, 48, 967-975. 
Landis J. R., \& Koch, G. G. (1977). The measurement of observer agreement for categorical data. Biometrics, 33, 159-174.

Levandowski, D. C., Koller, S. H., \& Piccinini, C. A. (2002). Paternidade na adolescência e os fatores de risco e de proteção para a violência na interação pai-criança. Interações, 7(13), 77-100.

Lewis, C., \& Dessen, M. A. (1999). O pai no contexto familiar. Psicologia: Teoria e Pesquisa, 15, 9-16.

Massola, G. M. \& Silvares, E. F. S. (2005). A percepção do distúrbio de comportamento infantil por agentes sociais versus encaminhamento para atendimento terapêutico. Revista Interamericana de Psicologia, 39, 139-150.

Mora, L., Otálora, C., \& Recagno-Puente, I. (2005). Hombre y la mujer frente al hijo: Diferentes voces sobre su significado. Psykhe, 14(2), 119-132.

Negreiros, T. C. G. M., \& Féres-Carneiro, T. (2004). Masculino e feminino na família contemporânea. Estudos e Pesquisas em Psicologia, 4(1), 34-47.

Phares, V. (1997). Accuracy of informants: Do parents think that mother knows best? Journal of Abnormal Child Psychology, 25, 165-171.

Piccinini, C. A., Silva, M. R., Gonçalves, T. R., Lopes, R. S., \& Tudge, J. (2004). O envolvimento paterno durante a gestação. Psicologia: Reflexão $e$ Crítica, 17, 303-314.

Programa Epi Info ${ }^{\mathrm{TM}}$ Version 3.3.2. Epi Info ${ }^{\mathrm{TM}}$. Recuperado em 25 setembro 2008, de http:// www.maurofontelles.med.br/06down.htm.

Rocha-Coutinho, M. L. (2003). O papel de homens e mulheres na família: Podemos falar em reestruturação? Psicologia Clínica, 15(2), 93-107.

Santos, E. O. L., \& Silvares, E. F. M. (2006). Crianças enuréticas e crianças encaminhadas para clínicas-escola: Um estudo comparativo da percepção de seus pais, Psicologia: Reflexão e Crítica, 19, 277-282.
Seiffge-Krenke, I., \& Kollmar, F. (1998). Discrepancies between mother's and fathers' perceptions of sons' and daughters' problem behavior: A longitudinal analysis of parent-adolescent agreement on internalising and externalising problem behavior. Journal of Child Psychology and Psychiatric, 39, 687-697.

Silvares, E. F. M., Meyer, S. B., Santos, E. O. L., \& Gerencer, T. T. (2006). Um estudo em cinco clínicas-escolas brasileiras com a Lista de Verificação Comportamental para Crianças [CBCL]. In: E. F. M. Silvares (2006). Atendimento psicológico em clínicas-escola (pp. 59-72). Campinas, SP: Editora Alíneas.

Soares, C., \& Sabóia, A. L. (2007). Tempo, trabalho e afazeres domésticos: Um estudo com base nos dados da Pesquisa Nacional por Amostra de Domicílios de 2001 e 2005. Textos para discussão. (Diretoria de Pesquisas, 21). Rio de Janeiro: IBGE. Recuperado em 25 novembro 2007, de http://www.ibge.gov.br/home/ estatistica/populacao/tempo_trabalho_afdom_ pnad2001_2005.pdf.

Treutler, C. M., \& Epkins, C. C. (2003). Are discrepancies among child, mother, and father reports on children's behavior related to parents' psychological symptoms and aspects of parent child relationships? Journal of Abnormal Child Psychology, 31, 13-27.

Wagner, A., Predebon, J., Mosmann, C., \& Verza, F. (2005). Compartilhar tarefas? Papéis e funções de pai e mãe na família contemporânea. Psicologia Teoria e Pesquisa, 21, 181-186.

Artigo recebido em 23/10/2007.

Aceito para publicação em 12/08/2008.

Derivado da dissertação de mestrado da primeira autora realizada entre os anos de 2006 e 2007, no grupo de pesquisa Formação, Avaliação e Atendimento em Psicoterapia Psicanalítica, sob orientação da segunda autora. Apoio: CNPq. 
Endereço para correspondência:

Juliane Callegaro Borsa. Rua Guilherme Alves, no. 901, apto. 404, Bairro Petrópolis. CEP 90680-001. Porto Alegre-RS, Brasil. E-mail: psicojuli@yahoo.com.br

Juliane Callegaro Borsa é doutoranda pelo Programa de Pós graduação em Psicologia da Universidade Federal do Rio Grande do Sul.

Maria Lucia Tiellet Nunes é Professor Titular da Faculdade de Psicologia da Pontifícia Universidade Católica do Rio Grande do Sul. 